\title{
Smart Check - COVID-19 triage system: evaluation of the impact on the screening time and identification of clinical manifestations of SARS-CoV-2 infection in a public health service.
}

\author{
Fernando Anschau ${ }^{1}$, Paulo Worm ${ }^{1}$, Luciane Kopittke ${ }^{1}$, Luis Humberto de Mello Villwock ${ }^{2}$, \\ Marcos Luiggi Lemos Sartori ${ }^{2}$, Sandro Pinheiro ${ }^{2}$, Jéferson Cardoso do Rosário², José \\ Francisco Secorun Inácio ${ }^{2}$, Maicon Diogo $\mathrm{Much}^{2}$, Eduardo Marckmann², João Ricardo \\ Nickenig Vissoci ${ }^{3}$, Wagner de Lara Machado ${ }^{2}$, Dalton Breno Costa ${ }^{2}$, Daniel Klug ${ }^{1}$, Juliane \\ Martin Prestes ${ }^{1}$, and Fabiano Hessel ${ }^{2}$ \\ ${ }^{1}$ Grupo Hospitalar Conceição \\ ${ }^{2}$ Pontifícia Universidade Católica do Rio Grande do Sul \\ ${ }^{3}$ Duke University
}

March 22, 2021

\begin{abstract}
Introduction: Most patients with COVID-19 have mild or moderate manifestations, however, there is a wide spectrum of clinical presentations and even more severe repercussions that require high diagnostic suspicion. Vital sign acquisition and monitoring are crucial for detecting and responding to patients with COVID-19. Objective: Thus, we conducted this study to demonstrate the impact of using a tool called Smart Check on the triage time of patients with suspected COVID-19 and to identify the main initial clinical manifestations in these cases. Methodology: We assessed triage times before and after the use of Smart Check in 11,466 patients. In this group, we identified 211 patients for the identification of COVID-19 clinical manifestations in a casecontrol analysis. Results: Smart Check was able to decrease the triage time by 33 seconds on average, with $75 \%$ of the exams being performed within 5 minutes, whereas with the usual protocol these steps were performed within 6 minutes. A range of clinical presentations made up the COVID-19 initial manifestations. Those with the highest frequency were dry cough (46.8\%), fever $(41.3 \%)$, dyspnea $(35.8 \%)$, and headache $(32.1 \%)$. Loss of appetite, fever, and ageusia were the manifestations that had a statistically significant association with the SARS-CoV-2 presence. Conclusions: Smart Check, a simple clinical evaluation tool, along with the targeted use of rapid PCR testing, can optimize triage time for patients with and without COVID-19. In triage centers, a number of initial signs and symptoms should be cause for SARS-CoV-2 infection suspicion, in particular the association of respiratory, neurological, and gastrointestinal manifestations. Keywords: new coronavirus, COVID-19, triage, clinical manifestations
\end{abstract}

Smart Check - COVID-19 triage system: evaluation of the impact on the screening time and identification of clinical manifestations of SARS-CoV-2 infection in a public health service.

Abstract:

Introduction: Most patients with COVID-19 have mild or moderate manifestations, however, there is a wide spectrum of clinical presentations and even more severe repercussions that require high diagnostic suspicion. Vital sign acquisition and monitoring are crucial for detecting and responding to patients with COVID-19. Objective: Thus, we conducted this study to demonstrate the impact of using a tool called Smart Check on the triage time of patients with suspected COVID-19 and to identify the main initial clinical manifestations in these cases. Methodology: We assessed triage times before and after the use of 
Smart Check in 11,466 patients. In this group, we identified 211 patients for the identification of COVID-19 clinical manifestations in a case-control analysis. Results: Smart Check was able to decrease the triage time by 33 seconds on average, with $75 \%$ of the exams being performed within 5 minutes, whereas with the usual protocol these steps were performed within 6 minutes. A range of clinical presentations made up the COVID-19 initial manifestations. Those with the highest frequency were dry cough (46.8\%), fever (41.3\%), dyspnea $(35.8 \%)$, and headache $(32.1 \%)$. Loss of appetite, fever, and ageusia were the manifestations that had a statistically significant association with the SARS-CoV-2 presence. Conclusions: Smart Check, a simple clinical evaluation tool, along with the targeted use of rapid PCR testing, can optimize triage time for patients with and without COVID-19. In triage centers, a number of initial signs and symptoms should be cause for SARS-CoV-2 infection suspicion, in particular the association of respiratory, neurological, and gastrointestinal manifestations.

Keywords: new coronavirus, COVID-19, triage, clinical manifestations

What's already known about this topic?

It is important to be assertive and quick in identifying cases of COVID-19;

The main signs and symptoms related to SARS-CoV-2 infection are referenced in the literature and exhibit a wide variety of clinical presentations.

What does this article add?

It is important to be assertive and quick in identifying cases of COVID-19 and the Smart Check tool can assist in this sorting.

The main signs and symptoms related to SARS-CoV-2 infection are referenced in the literature and exhibit a wide variety of clinical presentations; here we bring the comparison of these initial signs and symptoms with those presented in an acute respiratory syndrome or cold without COVID-19.

\section{Introduction:}

A new coronavirus $(\mathrm{CoV})$ with a high virulence and capable of infecting humans $(\mathrm{HCoV})$ is currently holding much of the world's population hostage. This virus, known as coronavirus 2, responsible for severe acute respiratory syndrome (SARS-CoV-2) and coronavirus 2019 (COVID-19) disease, emerged in late 2019 in China and is currently affecting the population in more than 210 countries and territories worldwide. On January 30, 2020, the WHO declared it a Public Health Emergency of International Concern and on April 11, 2020 it was declared a pandemic (1). At the time of this article's writing, the WHO estimates that COVID-19 has been diagnosed in 108,579,352 people from 210 countries worldwide, causing about 2,396,408 deaths (2). In Brazil there have been more than 9.8 million cases and about 240,000 deaths (2).

SARS-CoV-2 belongs to a family of viruses that can cause various symptoms such as pneumonia, fever, difficulty breathing, and pneumonitis (3). Clinical manifestations include fever, cough, dyspnea, myalgia, fatigue, headache, diarrhea, hemoptysis, anosmia, and augesia $(4,5)$. Most patients with COVID-19 have mild to moderate symptoms, but approximately $15 \%$ may progress to critical pneumonia and eventually develop severe acute respiratory syndrome (SARS), septic shock, multiple organ failure, and death (4). Once the infection sets in, it requires a high degree of suspicion for a correct diagnosis and the institution of appropriate therapeutic and restrictive measures due to the enormous manifestations spectrum.

Vital sign acquisition and monitoring are crucial for detecting and responding to deteriorating patients, however it is known that during routine vital sign acquisition and recording, many of the vital sign records in medical records are incomplete or inconsistent, which can compromise patient safety (6). Thus, we propose this study to demonstrate the impact of using a tool called Smart Check on the triage time of patients with suspected COVID-19 and identify its main clinical manifestations.

Methodology: 
We performed data collection of the time of risk classification and signs and symptoms of suspected COVID19 patients at the COVID-19 Triage Center of the Hospital Nossa Senhora da Conceição (HNSC) in Porto Alegre, in the southern region of Brazil, from June 1 to July 31, 2020. This triage center has a reception and waiting area, a registration and triage area, two boxes for exam collection, three consulting rooms, a stabilization room, and a specific environment for the professionals' paramentation and de-paramentation. It is open daily, from $8 \mathrm{am}$ to $10 \mathrm{pm}$, with a team of one administrative assistant, three nurses, three doctors, and four nursing technicians. In addition, it has three professional hygienists strategically distributed to keep the environment constantly disinfected. The COVID-19 triage center sees cases of influenza syndrome and identifies patients with potential symptoms for COVID-19, referring those with severity signs to the HNSC emergency room. The time to check vital signs (temperature, blood pressure, blood saturation, heart and respiratory rates) was considered as a classification time (table 1). The clinical manifestations were collected in anamnesis and categorized as listed in table 2.

In the period from July 1 to July 31, 2020, we introduced the Smart Check tool (multiparametric monitor from the company Toth Life Care ß; certificate of conformity TÜV 17.1492 dated 04/24/2018), a compact multiparametric vital signs monitor, into that triage center's care routine. Through Smart Check there is the possibility to quickly acquire vital signs (systolic, diastolic, and non-invasive mean arterial pressure and pulse rate, functional oxygen saturation, body temperature, blood glucose level) and, in triage mode, store and display the history of the triages performed. This triage tool communicates with external devices viaBluetooth, it has a bar code reader that allows quick identification of patients wearing identification bracelets, andethernet and wireless communication with transmission in HL7 protocol for integration with Hospital Information Systems. In this study, we used accessory Risk Classification software (SMART RISK@) for Emergency management.

To determine the impact of Smart Check on COVID-19 triage time, we conducted a before-and-after type study (n:11,466). To identify the characteristic clinical manifestations of COVID-19, we developed a descriptive study and a case-control analysis, where all patients (n:211), listed at random, who were included underwent real-time polymerase chain reaction (RT-PCR) testing of nasopharyngeal swab specimens. Nasopharyngeal swab samples in a single tube of viral transport medium were obtained under transmission-based precautions from all patients presenting to the triage center and comprised the descriptive case-control type study. All biological samples were sealed and transferred to the laboratory in strict accordance with standard protocol.

This study was approved by the Research Ethics Committee of the Grupo Hospitalar Conceição according to opinion $\mathrm{n}^{\circ} .3,968,873$ on April 14, 2020 and by the National Research Ethics Commission of Brazil through opinion $\mathrm{n}^{\circ} .3,990,822$ on April 26, 2020.

Considering an expected sensitivity of $95 \%$, expected specificity of $80 \%$, with a margin of error of $10 \%$ and a prevalence of $5.8 \%$ of COVID-19 in our population, our sample would be estimated at 210 individuals.

Statistical analysis:

We expressed continuous variables as medians and interquartile ranges or simple intervals, as appropriate. We summarized categorical variables as counts and percentages, and we calculated associations with Student's t-test, chi-square, and Mann-Whitney tests. We made no imputation for missing data. We performed all analyses using SPSS 22.0.

Results:

For the Smart Check tool time analysis, we collected data from 11,466 patients in triage for COVID-19. The group's average age was 41.4 years (18 to 98 years) and $58.3 \%$ were women. 2,258 (19.7\%) had some comorbidity, among which hypertension (45.8\%), diabetes mellitus (20.8\%), asthma (20\%), and heart disease $(6.2 \%)$ were the most frequently reported, followed by obesity and dyslipidemia (4.7\%), arthritis (4.3\%), smoking (4.2\%), alcoholism (4.2\%), and hypothyroidism (3.9\%). The average time from symptoms to seeking the COVID triage center was 4.4 days, with $64 \%$ of cases seeking care within 3 days of the onset of complaints. 
In table 1 we identified 4,799 patients who underwent COVID-19 triage in the usual manner with a mean time to risk classification of 4.3 minutes ( 6 minutes at the 75 th percentile). On the other hand, while using the Smart Check with 6,667 patients, the rating time was 3.74 minutes on average $(5$ minutes at the 75 th percentile).

We performed an analysis of 211 patients, listed at random, who had diagnostic testing for COVID-19 (RT-PCR by nasopharyngeal swab) to identify the clinical manifestations that led them to seek triage for COVID-19. In table 2, we show these signs and symptoms and their relationship to the presence of SARSCoV-2 infection. Dry cough (51 cases $(46.8 \%))$, fever (45 cases $(41.3 \%))$, dyspnea (39 cases (35.8\%)), and headache $(35$ cases $(32.1 \%)$ ) were the most frequent signs and symptoms. Loss of appetite, fever, and ageusia (loss of taste) were the manifestations that formed a statistically significant association with the presence of SARS-CoV-2. Dyspnea presented a p value of 0.07 and, when in conjunction with any of these last 3 clinical manifestations, it has shown an association with the presence of SARS-CoV-2 infection (table 3).

Discussion:

One strategy for fighting the COVID-19 pandemic was rapid diagnosis and care in these specific environments, with segregation of patients with the disease's most severe and characteristic symptoms. Even in such environments, patients who seek care for suspected infection may be exposed to the infection itself if they stay longer than necessary in triage centers. Nosocomial transmission of COVID-19 puts patients with other medical problems at risk of serious illness and death. In this study, we observed that $19.7 \%$ of patients with suspected COVID-19 had some comorbidity, which potentially put them at greater risk for serious illness.

Wake RM et al. demonstrated that from the total of nosocomial SARS-CoV-2 infections, $88 \%$ of patients had shared a ward with a confirmed COVID-19 case. In that study, the implementation of a triage system combining clinical evaluation with rapid testing for SARS-CoV-2 facilitated the cohort so that fewer susceptible patients were exposed to COVID-19 in shared environments. With the possibility of future waves of COVID-19-related hospitalizations, strategies to prevent nosocomial transmission are essential (7). With the use of the Smart Check screening tool there was an average reduction of 33 seconds in the time it took to classify patients with respiratory syndrome. Considering the 75th percentile of the classification time, we can observe a reduction of 1 minute in it. Point-of-care diagnostics can complement clinical evaluation to quickly identify patients with COVID-19 and reduce the risk of transmission within triage centers and hospitals.

If we take the decrease in the average time for classification with Smart Check and assess the number of cases classified in the month of use - July 2020 - it is possible to state that there was a reduction of 62 hours in the classification time/month. Thus, we can state that the Smart Check tool can contribute to the classification of patients with suspected Covid-19, bringing celerity to this screening moment. Since Smart Check quickly characterizes patients' signs and symptoms with accurate records, we can reduce the risk of transmission and profile clinical manifestations.

Most patients with COVID-19 exhibit mild to moderate symptoms, but approximately $15 \%$ progress to critical pneumonia and 5\% eventually develop acute respiratory distress syndrome, multiple organ failure, septic shock, and death $(4,8)$. Once the infection sets in, the spectrum of clinical presentations ranges from asymptomatic infection to critical respiratory failure. Most commonly reported symptoms are fever, cough, myalgia, fatigue, pneumonia, dyspnea, as well as loss of smell and taste, while less commonly reported symptoms include diarrhea, hemoptysis, and coryza $(4,5)$.

We found a range of clinical manifestations that comprised the diagnosis of SARS-Cov-2 infection, among which dry cough $(46.8 \%$ of cases), fever $(41.3 \%)$, dyspnea $(35.8 \%)$, and headache $(32.1 \%)$ were the most frequent. However, this clinical presentation is similar to other respiratory diseases such as influenza, which makes us need some degree of suspicion for the COVID-19 diagnosis. Since the clinical manifestations were collected by direct anamnesis at the time of screening, we compete with the risk of measurement bias. Patients, subjects of the research, could not remember related signs or symptoms or overestimate those who would have them perform COVID-19 screening tests. Fever seems to be one of the initial events, after 2 to 3 days of infection, followed by pulmonary manifestations (9). Loss of appetite, fever, and ageusia (loss of taste) 
were the manifestations that formed a statistically significant association with the presence of SARS-CoV-2 in our study. Dyspnea, when in conjunction with loss of appetite, fever, or ageusia, has also shown an association with the presence of SARS-CoV-2 infection. The presence of more than one symptom, especially when we identify the search for care after 3 days of symptoms, has been pointed out as characteristic of COVID-19, when we can observe, besides respiratory manifestations (dyspnea, cough), some neurological manifestations such as ageusia, fatigue, anosmia, headache and myalgia, and other gastrointestinal manifestations such as loss of appetite, diarrhea, and abdominal pain (10).

Conclusion:

While social distancing measures can reduce the transmission of COVID-19 in the community, this transmission in triage centers and hospitals continues to put vulnerable populations at risk of more serious illness and death. As we identify a new wave of infections and hospital admissions, prevention of nosocomial transmission remains vitally important. We demonstrated how a simple clinical evaluation tool - the Smart Check - along with the targeted use of rapid PCR testing, can optimize triage time for patients with and without COVID-19. In addition, a number of early signs and symptoms should be cause for suspicion of SARS-CoV-2 infection, in particular the association of respiratory, neurological, and gastrointestinal manifestations.

References:

1. Cucinotta D, Vanelli M. WHO declares COVID-19 a pandemic. Acta Biomed 2020;91:157-160.

2. World Health Organization. Coronavirus disease 2019 (COVID-19) Situation Report - 98. 2020 [cited 2021 Feb 11]. https://www. who. int/ emergencies/ diseases/ novel- coronavirus- 2019/situationreports/.

3. WMHC. Wuhan Municipal Health and Health Commission's Briefingon the Current Pneumonia Epidemic Situation in Our City. 2020.http:// wjw. wuhan. gov. cn/ front/ web/ showDetail/ 2019123108989. Accessed 1 Feb 2021.

4. Xu Z, Shi L, Wang Y, Zhang J, Huang L, Zhang C, et al. Pathological findings of COVID-19 associated with acute respiratory distress syndrome. Lancet Respir Med (2020) 8:420-2. doi: 10.1016/S22132600(20)30076-X.

5. He F, Deng Y, Li W. Coronavirus Disease 2019 (COVID-19): What we know? J Med Virol (2020) 92:719-25. doi: 10.1002/jmv.25766.

6. Stevenson JE, Israelsson J, Nilsson GC, Petersson GI, Bath PA. Recording signs of deterioration in acute patients: The documentation of vital signs within electronic health records in patients who suffered in-hospital cardiac arrest. Health Informatics J. 2016;22(1):21-33.

7. Wake RM, Morgan M, Choi J, Winn S. Reducing nosocomial transmission of COVID-19: implementation of a COVID-19 triage system. Clin Med (Lond). 2020 Sep;20(5):e141-e145. doi: 10.7861/clinmed.2020-0411. Epub 2020 Aug 11. PMID: 32788160; PMCID: PMC7539706.

8. Huang C, Wang Y, Li X, Ren L, Zhao J, Hu Y, et al. Clinical features of patients infected with 2019 novel coronavirus in Wuhan, China. Lancet (2020) 395:497-506. doi: 10.1016/S0140-6736(20)30183-5.

9. Awadasseid A, Wu Y, Tanaka Y, Zhang W. Initial success in the identification and management of the coronavirus disease 2019 (COVID-19) indicates human-to-human transmission in Wuhan, China. Int J Biol Sci . 2020;16(11):1846-1860. Published 2020 Apr 6. doi:10.7150/ijbs.45018.

10. Hu B, Guo H, Zhou P, Shi ZL. Characteristics of SARS-CoV-2 and COVID-19 [published online ahead of print, 2020 Oct 6]. Nat Rev Microbiol . 2020;1-14. doi:10.1038/s41579-020-00459-7.

\section{Hosted file}

Table 1.pdf available at https://authorea.com/users/403237/articles/514787-smart-
check-covid-19-triage-system-evaluation-of-the-impact-on-the-screening-time-and-
identification-of-clinical-manifestations-of-sars-cov-2-infection-in-a-public-health-
service

\section{Hosted file}


Table 2.pdf available at https://authorea.com/users/403237/articles/514787-smartcheck-covid-19-triage-system-evaluation-of-the-impact-on-the-screening-time-andidentification-of-clinical-manifestations-of-sars-cov-2-infection-in-a-public-healthservice

\section{Hosted file}

Table 3.pdf available at https://authorea.com/users/403237/articles/514787-smartcheck-covid-19-triage-system-evaluation-of-the-impact-on-the-screening-time-andidentification-of-clinical-manifestations-of-sars-cov-2-infection-in-a-public-healthservice 